\title{
MODELAGEM GEOESTATÍSTICA DA OCORRÊNCIA DE CASOS DE LEPTOSPIROSE E ALAGAMENTOS NO MUNICIPIO DE CURITIBA - PR, NO ANO DE 2014
}

\author{
B. DOZSA, M. L. C. D. MONEGO* e L. KUMMER
}

Universidade Tecnológica Federal do Paraná (UTFPR) - Departamento Acadêmico de Química e Biologia mauriciluzia@gmail.com*

Artigo submetido em outubro/2015 e aceito em janeiro/2016

DOI: $10.15628 /$ holos. 2016.3857

\section{RESUMO}

O objetivo deste estudo foi aplicar a metodologia geoestatística para avaliar os casos de leptospirose no município de Curitiba, relacionando-os com as inundações ocorridas na cidade no mesmo período. Após uma análise descritiva para verificar a existência de dependência espacial, construiu-se os variogramas experimentais que foram ajustados pelos modelos esférico, exponencial e gaussiano. Os mapas krigados mostraram claramente o comportamento da doença e das inundações na área de estudo, fornecendo informações importantes para o gerenciamento dos riscos.

PALAVRAS-CHAVE: Geostatística, mapeamento, leptospirose, alagamentos.

\section{GEOSTATISTICAL MODELING OF THE OCCURRENCE LEPTOSPIROSIS CASES AND FLOODING IN CURITIBA - PR CITY, IN 2014}

\section{ABSTRACT}

The purpose of this study was to analyze the applicability of geostatistics for modeling information for the assessment of cases of leptospirosis and flooding in the city of Curitiba. A descriptive analysis to verify the existence of spatial dependence was done and experimental semivariogras were built fitting three theoretical models: spherical, exponential and gaussian The kriged maps show clearly the spatial variation of the disease analyzed in the study area, providing important information for managing risks.

KEYWORDS: Geostatistical mapping, Environmental epidemiology, Flooding. 


\section{INTRODUÇÃO}

O quadro epidemiológico no Brasil caracteriza-se pela coexistência de doenças degenerativas e o retorno de antigas doenças infecciosas, dentre elas a leptospirose, que desafiam radicalmente a saúde pública do século XXI na busca de soluções (Albuquerque e Morais, 1997).

Considerada uma zoonose de importância mundial, causada pela espiroqueta patogênica do gênero Leptospira, transmitida pelo contato com urina de animais infectados ou água e lama contaminadas pela bactéria, a leptospirose é um significativo problema para saúde pública em países tropicais em desenvolvimento (Lima, 2009). Isto ocorre devido à alta incidência nas populações que vivem em aglomerações urbanas sem a adequada infraestrutura sanitária e com altas infestações de roedores. Estes fatores, associados às estações chuvosas e às inundações, fazem do Brasil um ambiente propício à ocorrência de leptospirose (Brasil, 2009).

Mesmo com tantos casos de epidemias, a leptospirose ainda não recebe a devida prioridade, pois há poucos trabalhos com propostas preventivas para diminuir o impacto da doença. Além disso, ainda não se tem muitos estudos que contribuem para compreender o perfil da população (que foge dos grupos de risco) atingida por essas epidemias (Tassinari, 2009).

No país, a doença tem grande importância social e econômica, devido à sua alta incidência, cus to hospitalar, perdas de dias de trabalho como também por sua letalidade (Bernardi, 2012). No meio urbano, os principais vetores para a persistência de focos de infecção são os roedores (especialmente o rato de esgoto), outros vetores são os suínos, bovinos, equinos, ovinos e cães (Sarkar, 2002). O homem, hospedeiro terminal e acidental da doença, infecta-se ao entrar em contato com a urina de animais infectados com a bactéria, de modo direto ou indireto, por meio do contato com água, lama ou solo contaminados. A penetração do microrganismo ocorre através da pele com lesões, mucosas e da pele íntegra quando imersa em água por longo tempo (Bernardi, 2012).

A disseminação e a persistência da Leptospira no ambiente predispõem o contato do homem com águas contaminadas e facilitam a ocorrência de surtos. Um dos principais padrões epidemiológicos da leptospirose encontrados no Brasil são epidemias urbanas anuais, principalmente em comunidades carentes, pós-enchentes e inundações (Brasil, 2009).

Análises dos dados históricos de alagamentos do município de Curitiba mostram que as bacias mais afetadas são as dos rios Barigui, Belém e Atuba, pois são as que possuem maior densidade de ocupação e áreas impermeabilizadas, bem como grande densidade populacional. Tais fatores em conjunto com precipitações intensas são os principais vetores que contribuem para a ocorrência de alagamentos (Lohmann, 2013).

Atualmente, é possível perceber que há uma preocupação com o meio ambiente, voltada a garantir uma maior qualidade de vida aos seres humanos. Pesquisas têm ajudado a desenvolver programas de monitoramento e avaliação de riscos envolvendo todos os setores do ambiente. Um maior entendimento dos fenômenos ambientais permite o desenvolvimento de métodos mais eficientes para a análise e tratamento de dados, desta forma, é possível se obter informações significativas dos fenômenos auxiliando a tomada de decisões (Monego, 2012). 
Uma análise de dados utilizando métodos estatísticos clássicos geralmente supõe que as realizações das variáveis aleatórias são independentes entre si. Observa-se, portanto, que os fenômenos naturais apresentam frequentemente com uma certa estruturação nas variações entre vizinhos, portanto, apresentam algum grau de dependência espacial (Campos, 2011).

Os métodos geoestatísticos vêm sendo largamente utilizados para avaliação de dados ambientais. Essa metodologia utiliza procedimentos estatísticos aplicados a situações cujos dados estão relacionados a fenômenos naturais espacialmente distribuídos e correlacionados (Druck et al, 2004).

Ao projetar trabalhos envolvendo o monitoramento ambiental o principal objetivo, em termos gerais, é informar o estado em que se encontra o meio ambiente. A proposta desse estudo foi investigar o emprego da metodologia geoestatística na avaliação das regiões de maior risco de casos confirmados de leptospirose, e das regiões de maior ocorrência de alagamento, no município de Curitiba, no ano de 2014. Os mapas gerados fornecerão informações relevantes auxiliando na avaliação do impacto ambiental gerado, colaborando nas ações de planejamento e gestão de saúde, urbana e ambiental do município.

\section{MATERIAL E MÉTODOS}

\section{1 Área de estudo}

Neste trabalho utilizamos a área do município de Curitiba juntamente com os conjuntos de dados de casos de Leptospirose, cedidos pela Secretaria Municipal de Saúde (SMS) do município de Curitiba e os conjuntos de dados de alagamentos que foram cedidos pela Coordenadoria Municipal de Proteção e Defesa Civil de Curitiba (COMPDEC) para a realização na análise. Foram usados dados de casos confirmados de Leptospirose do ano de 2014, bem como os casos de alagamento do referido ano.

\subsection{Análise geoestatística}

Para a análise geoestatística, tanto dos casos confirmados de leptospirose, quanto dos casos registrados de alagamentos, foram seguidas as etapas conforme a Figura 1.

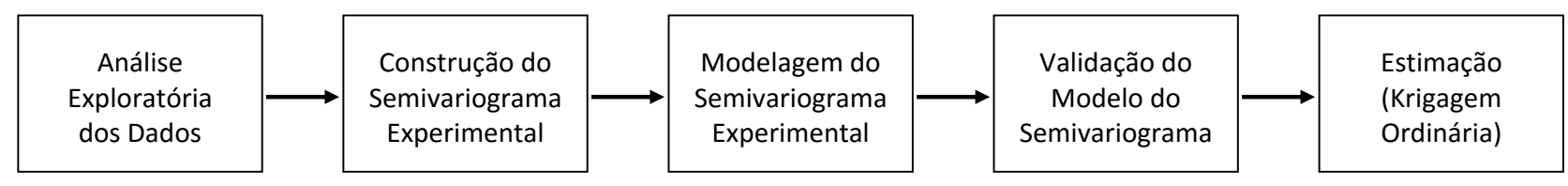

Figura 1: Etapas utilizadas na realização da análise geoestatística do conjunto de dados confirmados de leptospirose e dos dados registrados de alagamentos do município de Curitiba no ano de 2014 .

Primeiramente, na análise exploratória, foi realizado um diagnóstico do conjunto de dados para identificar a presença de dependência espacial, ocorrência de valores anômalos, existência de assimetrias na distribuição e a eventual presença de tendências. Em seguida nas etapas 2 e 3 , foram construídos e modelados os semivariogramas experimentais dos dois conjuntos de dados, permitindo quantificar a dependência especial. Na etapa 4, foi realizada a validação cruzada, que permitiu através de técnicas estatísticas escolher o modelo que melhor se ajustasse aos dados 
analisados. Finalmente, após escolhido o modelo, na etapa 5, os dados foram estimados por krigagem.

\subsection{Análise exploratória de dados}

Nas análises exploratórias dos dados foi utilizado o Google Earth para se obter as coordenadas geográficas (latitudes e longitudes) de cada bairro, bem como a delimitação do polígono do município de Curitiba. Também foram feitas planilhas com tabelas no Micros oft Excel ${ }^{\circledR}$ que continham as coordenadas geográficas e o número de casos para cada bairro no ano. A segunda etapa da análise consistiu em criar um roteiro e utilizá-lo para analisar os dados através do software R e pacote geoR (R Development Core Team, 2006; Ribeiro Jr. e Diggle, 2001).

\subsection{Construção e Modelagem do Semivariograma Experimental}

A etapa de construção do semivariograma experimental é de extrema importância na metodologia geoestatística, pois é neste ponto que se faz a síntese das características estruturais do fenômeno espacial em um modelo único e coerente. A equação de Matheron para o semivariograma experimental está representada na Equação 1.

$$
\gamma(h)=\frac{1}{2 N(h)} \sum_{i=1}^{N(h)}\left[Z\left(x_{i}\right)-Z\left(x_{i}+h\right)\right]^{2}
$$

onde $Z\left(x_{i}\right)$ é o valor da variável de interesse na localização $\left(x_{i}\right)$ e $N(h)$ é o número de pares de pontos separados pelo vetor lag $(h)$. Nesse cálculo à medida que se aumenta a dimensão de $h$, menor é a área representativa dos pares de pontos, assim não se deve exceder valores de $h$ superiores a aproximadamente metade da maior distância entre as observações (Soares, 2000).

Dentre os diversos modelos de ajuste, os mais utilizados são o modelo es férico, exponencial e gaussiano que normalmente cobrem a generalidade das situações de dispersão de fenômenos espaciais nas ciências da terra e do ambiente (Soares, 2000). Os modelos estão representados, respectivamente, nas Equações 2, 3 e 4.

$$
\begin{gathered}
\gamma(\mathrm{h})= \begin{cases}\mathrm{C}_{0}+\mathrm{C}_{1}\left[\frac{3}{2}\left(\frac{\|\mathrm{h}\|}{\mathrm{a}}\right)-\frac{1}{2}\left(\frac{\|\mathrm{h}\|}{\mathrm{a}}\right)^{3}\right], & 0 \leq \mathrm{h}<\mathrm{a} \\
\mathrm{C}_{0}+\mathrm{C}_{1}, \quad \mathrm{~h} \geq \mathrm{a}\end{cases} \\
\gamma(\mathrm{h})= \begin{cases}\mathrm{C}_{0}+\mathrm{C}_{1}\left[1-\exp \left(-\frac{3\|\mathrm{~h}\|}{\mathrm{a}}\right)\right], & \mathrm{h} \geq 0\end{cases} \\
\gamma(\mathrm{h})= \begin{cases}\mathrm{C}_{0}+\mathrm{C}_{1}\left[1-\exp \left(-3\left(\frac{\|\mathrm{h}\|}{\mathrm{a}}\right)^{2}\right)\right], & \mathrm{h} \geq 0\end{cases}
\end{gathered}
$$


Nas equações, três parâmetros são importantes. O alcance (a) que é a distância na qual o valor da semivariância se torna constante e, assim, pode-se estabelecer um ponto de corte (inexistência de correlação entre as medidas). O patamar $\left(C_{1}+C_{0}\right)$, que representa a variância estruturada corresponde ao valor máximo das semivariâncias, ou seja, é o valor da semivariância quando $h=$ a. Outro parâmetro importante é o efeito pepita ou nugget $\left(C_{0}\right)$, são erros de medição ou variabilidade pequena, medidas próximas possuem valores diferentes. Com isso, temos um valor não nulo de semivariância que deve ser incluído no modelo do semivariograma.

A característica do semivariograma observada é que os valores das semivariâncias aumentam à medida que aumenta a distância entre as medidas (Isaaks, 1989).

Neste trabalho utilizamos, também, como modelo de semivariograma, o modelo Matérn, que foi proposto por Diggle e Ribeiro Junior (2000).

A família dos modelos de Matérn é definida na equação 5.

$$
\rho(h, a, k)=\left[2^{k-1} \Gamma(k)\right]^{-1}\left(\frac{h}{a}\right)^{k} K_{k}\left(\frac{h}{a}\right)
$$

onde $\rho(h)$ representa a correlação entre pares de pontos separados pela distância $h$; (a) é o parâmetro de alcance do modelo; $\Gamma(k)$ é a função Gama e $K_{k}$ é a função Bessel modificada de ordem $k$.

Esta função apresenta um parâmetro $k$ chamado de ordem do modelo Matérn o qual determina a suavização analítica num modelo Gaussiano. Para $k=0,5$ o modelo Matérn reduz-se ao modelo exponencial e para $k$ tendendo a infinito, equivale ao modelo gaussiano. Isso permite maior flexibilidade para caracterizar a variabilidade do variograma experimental. Os autores do modelo concluíram que a família Matérn é provavelmente a melhor escolha quanto uma função de correlação somente com dois parâmetros para uso generalizado (Oliveira, 2003).

O grau de dependência espacial da variável em es tudo pode ser avaliado através da razão efeito pepita/patamar. Se o valor for inferior a $25 \%$, sugere que a variável possui forte dependência es pacial. Um valor entre $25 \%$ e $75 \%$ sugere que a dependência é moderada. Caso o valor ultrapasse $75 \%$, a dependência espacial é fraca (Wei, 2007).

\subsection{Validação do Modelo do Semivariograma}

Foi utilizada a técnica de validação cruzada, para efeito de avaliação e/ou comparação do desempenho dos modelos e métodos ajustes de semivariograma. Nesta técnica o processo de krigagem é usado para estimar o valor do parâmetro em estudo nos pontos amostrados. Durante o processo, cada uma das observações é temporariamente retirada do conjunto de dados e o seu valor é estimado utilizando as observações restantes, repetindo-o para todos os pontos amostrais. Os valores estimados e os valores reais podem ser comparados usando uma mesma análise estatística para esses dois conjuntos de dados e/ou efetuando uma análise estatística dos erros. $\mathrm{Na}$ análise estatística dos erros são calculados os indicadores estatísticos: erro médio (EM), raiz quadrada do erro quadrático médio (REQM) e Erro Quadrático Estandardizado Médio (EQEM), respectivamente nas Equações 6, 7 e 8 (Wackernagel, 2003). 


$$
\begin{gathered}
E M=\frac{1}{m} \sum_{i=1}^{m}\left[Z\left(x_{i}\right)-\hat{Z}\left(x_{i}\right)\right] \\
R E Q M=\sqrt{\frac{1}{m} \sum_{i=1}^{m}\left[Z\left(x_{i}\right)-\hat{Z}\left(x_{i}\right)\right]^{2}} \\
E Q E M=\frac{1}{m} \sum_{i=1}^{m} \frac{\left[Z\left(x_{i}\right)-\hat{Z}\left(x_{i}\right)\right]^{2}}{\alpha_{K(x)_{i}}^{2}}
\end{gathered}
$$

onde: $Z\left(x_{i}\right)$ e $\hat{Z}\left(x_{i}\right)$ são, respectivamente, a medida e a estimativa no ponto $x i, m$ é o número de medidas do conjunto de validação e $\alpha_{K(x)_{i}}^{2}$ é a variância da krigagem no ponto estimado.

\subsection{Krigagem Ordinária}

O método geoestatístico estimado krigagem leva em consideração as características espaciais de autocorrelação de variáveis regionalizadas, elas devem possuir uma certa continuidade espacial, o que permite que os dados obtidos por amostragem de certos pontos possam ser usados para parametrizar a estimação de pontos onde o valor da variável seja desconhecido. A verificação da existência de continuidade espacial é feita por meio da análise do variograma que determinará os parâmetros que caracterizam o comportamento regionalizado. Utiliza-se distâncias ponderadas e estimativas por médias móveis, pelo qual os pesos adequados são obtidos a partir de um variograma representativo da média das diferenças ao quadrado dos valores de $Z$ (xi) distribuídos em intervalos de distâncias especificados (lags $h$ ).

O sistema de krigagem necessário para a determinação dos ponderadores associados a cada um dos pontos estimadores baseia-se na ideia que quanto maior a covariância entre uma amostra $x i, i=1,2, \ldots, n$, e o local que está sendo estimado, $x_{0}$, mais essa amostra deve contribuir para a estimativa (Landim, 2002). A equação da krigagem está representado na Equação 9.

$$
\hat{Z}\left(x_{0}\right)=\sum_{i=1}^{M} \alpha_{i} Z\left(x_{i}\right)
$$

onde $Z\left(x_{i}\right), \mathrm{i}=1,2, \ldots, \mathrm{M}$ são as observações próximas à $x_{0}$ (ponto a estimar) e $\alpha_{i}, \mathrm{i}=1,2, \ldots, \mathrm{M}$ os pesos respectivos. A Equação 10 indica a variância de krigagem, $\sigma^{2}\left(x_{i}\right)$ (Soares, 2000).

$$
\sigma^{2}\left(x_{i}\right)=\mathrm{E}\left[\left(\hat{Z}\left(x_{0}\right)-Z\left(x_{0}\right)\right)^{2}\right]=\sum_{i=1}^{M} \alpha_{i} \gamma\left(x_{i,} x_{0}\right)-\lambda
$$

onde $\lambda$ é o parâmetro de Lagrange, usado para cumprir a condição de não-enviesamento (soma dos pesos unitária - $\sum_{\mathrm{i}=1}^{\mathrm{M}} \alpha_{\mathrm{i}}=1$ ). 
A krigagem ordinária procura que a média dos erros de estimação seja nula e tenta minimizar a variância dos erros de estimação, sendo estes os pressupostos fundamentais do método.

\section{RESULTADOS E DISCUSSÕES}

Na Figura 2 (esquerda) podem ser observados os bairros do município de Curitiba que apresentaram o maior número de casos confirmados de leptospirose no ano de 2014. Os bairros localizados na região central do município foram os mais afetados. Os pontos em verde e amarelo, que representam entre 3 a 9 casos encontram-se nessa região central. Ao sul do município somente em dois bairros foram detectados mais de 3 casos.
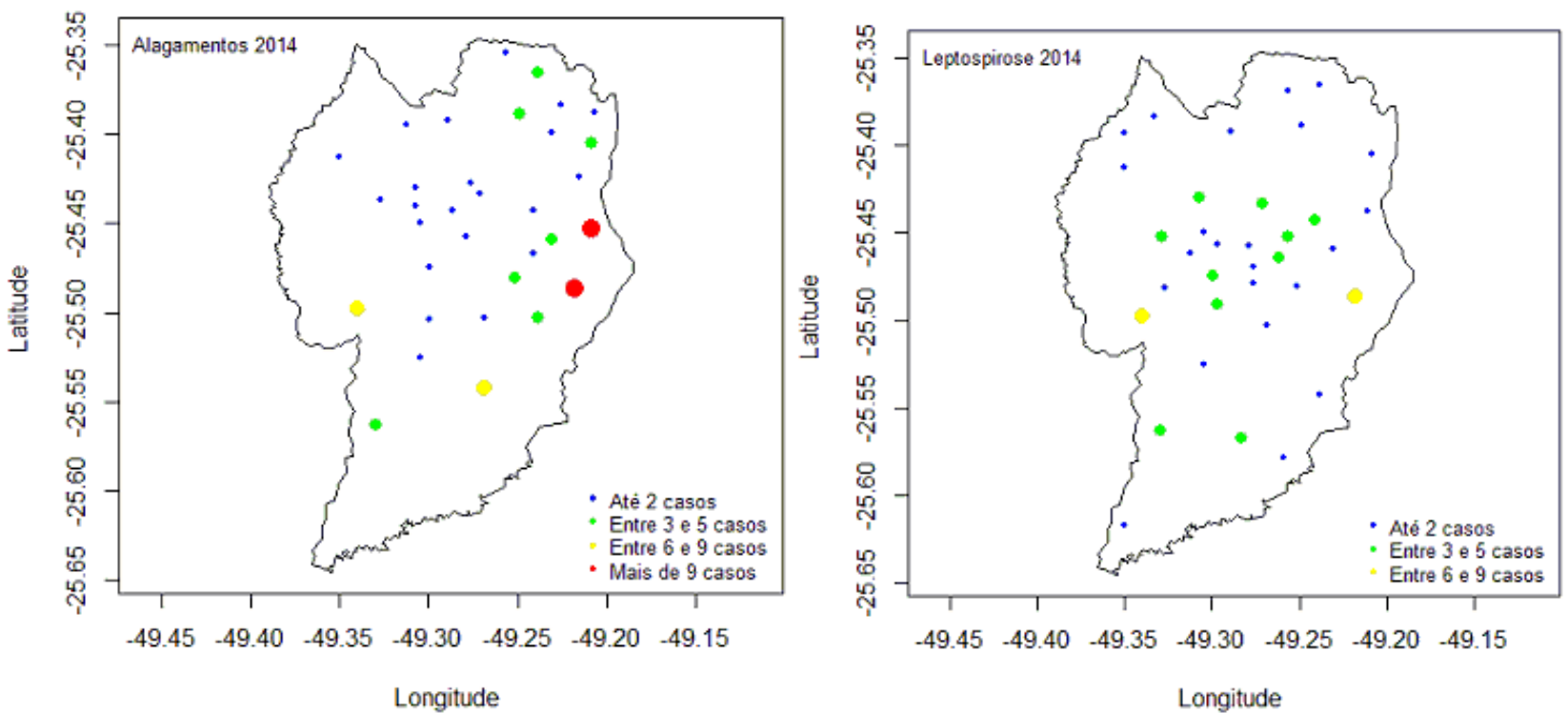

Figura 2: Representação bidimensional dos casos confirmados de leptospirose (esquerda) e dos casos registrados de alagamentos (direita) no ano de 2014, localizados no município de Curitiba.

Os bairros onde foram registrados casos de alagamentos, no mesmo ano, estão concentrados mais a oeste do município. Na Figura 2 (direita) podem ser observados que os pontos em amarelo, verde e vermelho, que representam acima de 3 casos encontram-se localizados mais a oeste do mapa, tendo somente um bairro a leste e um ao sul.

\subsection{Análise Exploratória e da Estrutura de Continuidade Espacial dos Dados}

Para dar início a análise geoestatistica dos dois conjuntos de dados realizou-se uma análise estatística descritiva para identificar a presença de dependência espacial, ocorrência de valores anômalos, existência de assimetrias na distribuição e a eventual presença de tendências.

As funções utilizadas na análise estão disponíveis e descritas no software estatístico $R$ no pacote geoR. Os resultados da análise estão apresentados na Tabela 1. Os valores para os coeficientes de assimetria foram positivos, o que nos indica uma assimetria positiva na distribuição. Os valores para o coeficiente de variação são interpretados como a variabilidade dos dados em relação à média, quanto menor o seu valor mais homogêneo é o conjunto de dados. Em nossas análises os valores se apresentaram altos, o que caracteriza uma certa heterogeneidade no conjunto de dados. 
Tabela 1: Sumário estatístico dos dados de leptospirose e alagamentos no ano de 2014.

\begin{tabular}{c|c|c}
\hline Parâmetros & Leptospirose & Alagamento \\
\hline Observações & 38 & 33 \\
\hline Mínimo & 1 & 1 \\
\hline Média & 2,47 & 3,03 \\
\hline Mediana & 2 & 1 \\
\hline Máximo & 9 & 20 \\
\hline Assimetria & 1,88 & 2,91 \\
\hline Curtose & 3,52 & 8,11 \\
\hline Variância & 3,93 & 17,47 \\
\hline Desvio padrão & 1,98 & 4,18 \\
\hline Coef. Variação (\%) & 80,15 & 137,92 \\
\hline
\end{tabular}

Esses resultados preliminares já apresentam indícios de que a endemia de leptospirose não possui um caráter homogêneo dentro do município. Acreditamos que não seja significativo, nesse caso, a preocupação com a média de casos confirmados anualmente, e sim onde se encontram a concentração do maior número de casos. Com esses resultados poderemos inferir sobre as causas e os fatores que podem ter levado a tais números.

Continuando a análise exploratória dos dados, foram elaborados os histogramas para o conjunto de dados confirmados de leptospirose (Figura 3 (esquerda)) e casos registrados de alagamentos (Figura 3(direita)), utilizando escalas individuais para facilitar a compreensão dos mesmos. Os histogramas confirmam o que foi observado nas análises anteriores, ou seja, há uma leve assimetria nos dois conjuntos de dados.
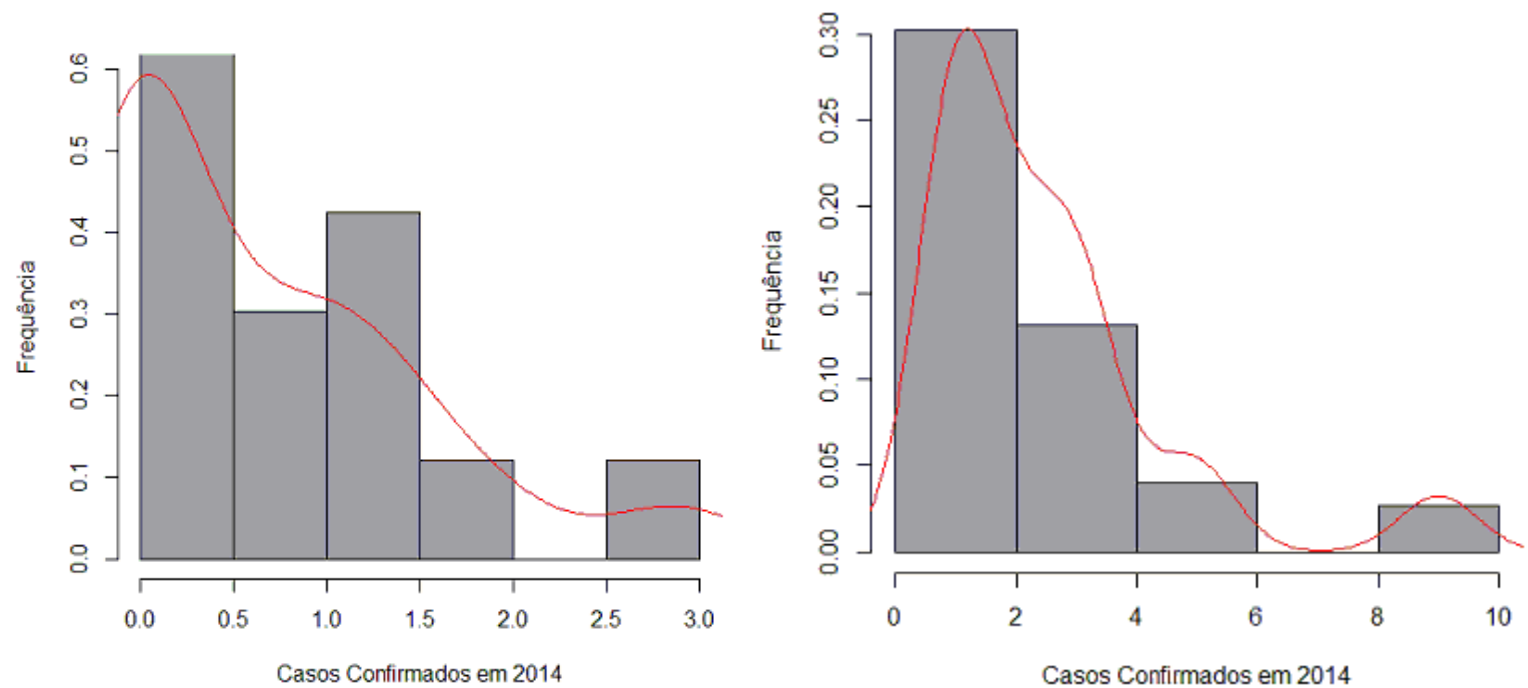

Figura 3: Histogramas dos conjuntos de dados para os casos confirmados de leptospirose (esquerda) e casos registrados de alagamentos (direita) do município de Curitiba no ano de 2014.

As estimativas realizadas utilizando a krigagem apresentam melhores resultados quando os dados seguem uma distribuição ao menos aproximadamente normal (Paz-Gonzalez, 2001). Como foi detectado um certo viés na distribuição, foi investigado o comportamento dos dados, quanto a normalidade, considerando-se transformações logarítmicas que não apresentaram alterações significativas, dessa forma se optou por utilizar os dados originais não transformados. 


\subsection{Semivariograma Experimental, Modelos e Métodos de Ajuste}

Uma outra forma de verificar a dependência espacialé através do semivariograma empírico ou experimental. Foram gerados os semivariogramas empíricos para os dados originais dos casos confirmados de leptospirose (Figura 4 (esquerda)) e casos registrados de alagamentos (Figura 4 (direita)) para o ano de 2014. Utilizou-se o modelo estacionário isotrópico, que calcula a distância em todas as direções. Estabeleceu-se o ponto de corte dos semivariogramas para os diferentes modelos através de uma análise visual, levando-se em consideração uma fração da máxima distância e a sua estabilização após uma certa distância. O ponto de corte dos semivariogramas não excedeu os valores de aproximadamente metade da maior distância entre as observações .

Foi verificada a presença de correlação espacial nitidamente para os dois conjuntos de dados, de forma geral a evidência de dependência espacial decresce com a distância. Para modelar a dependência espacial testamos os modelos esférico, exponencial, gaussiano, e a família Matérn de funções de correlação.
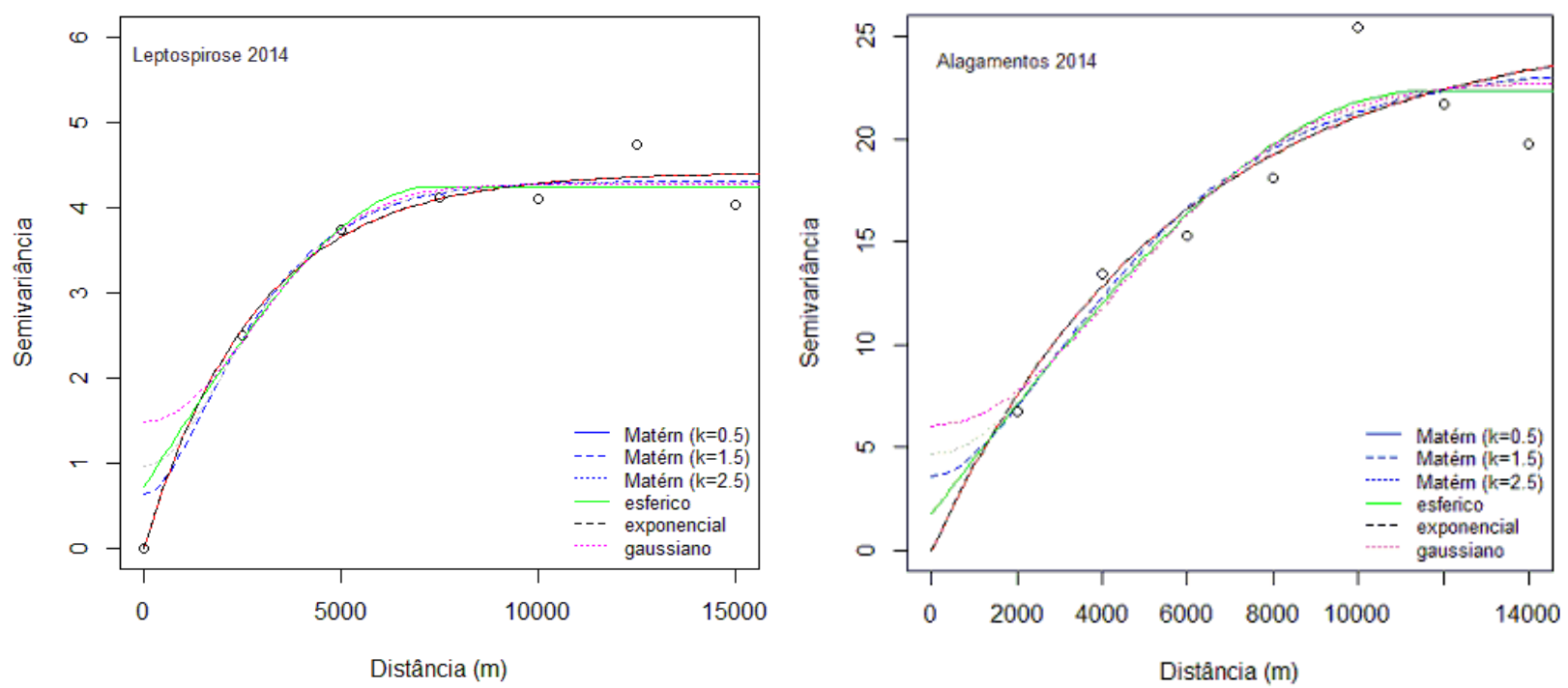

Figura 4: Semivariograma empírico e modelos ajustados para os casos confirmados de leptospirose (esquerda) e casos registrados de alagamentos (direita) do município de Curitiba no ano de 2014.

Optamos por utilizar a família Matérn uma vez que, devido ao parâmetro de suavização incorporado na função, adaptar-se melhor, produzindo ajustes mais adequados à estrutura de correlação dos dados espaciais. Na análise visual dos semivariogramas, para todos os modelos, observou-se o crescimento a partir da origem. A Tabela 2 mostra os parâmetros dos modelos analisados. O modelo esférico apresentou um alcance maior em relação aos outros modelos testados, e esse comportamento pode ser observado, tanto para os dados de leptospirose quanto de alagamentos. Os valores do parâmetro $C_{1}$ mostram que a variabilidade dos dados é muito similar. 
Tabela 2: Parâmetros dos modelos para os dados de leptospirose e alagamentos no ano de 2014.

\begin{tabular}{c|c|c|c|c|c|c}
\hline \multirow{2}{*}{ Model os } & \multicolumn{3}{|c|}{ Leptospirose } & \multicolumn{3}{c}{ Alagamentos } \\
\cline { 2 - 7 } & $\mathrm{C}_{0}$ & $\mathrm{C}_{1}$ & $\mathrm{a}$ & $\mathrm{C}_{0}$ & $\mathrm{C}_{1}$ & $\mathrm{a}$ \\
\hline Matérn $k=0,5$ & 0 & 4,17 & 2842,01 & 0 & 25,57 & 5736,10 \\
\hline Matérn $k=1,5$ & 0,63 & 3,69 & 1499,96 & 3,59 & 20,06 & 2701,53 \\
\hline Matérn $k=2,5$ & 0,96 & 3,34 & 1183,24 & 4,68 & 18,68 & 2050,69 \\
\hline Esférico & 0,72 & 3,52 & 7336,47 & 1,80 & 20,54 & 11559,43 \\
\hline Exponencial & 0 & 4,42 & 2842,01 & 0 & 25,57 & 5736,11 \\
\hline Gaussiano & 1,48 & 2,79 & 3869,74 & 6,05 & 16,76 & 6168,44 \\
\hline
\end{tabular}

Como mencionado, é importante avaliar os modelos testados para escolher o que melhor represente a estrutura espacial do conjunto de dados. Os modelos foram avaliados através dos indicadores da validação cruzada. Se a média dos erros da validação cruzada (ou Erro Médio (EM)) estiver próxima de 0 , pode-se dizer que, aparentemente, as estimativas são não enviesadas. 0 valor de REQM deve ser o menor possível, indicando que as estimativas estão próximas das medidas. A média dos erros de validação cruzada quadráticos estandardizados (ou Erro Quadrático Estandardizado Médio (EQEM)) deve ser próximo de um, indicando que o modelo é satisfatório. Os indicadores da validação cruzada informados na Tabela 3, para os dois conjuntos de dados, são muito próximos, mas para os modelos Matérn $k=0,5$ e exponencial, apresentam-se relativamente melhores.

Tabela 3: Indicadores da validação cruzada para os para os dados de leptospirose e alagamentos no ano de 2014.

\begin{tabular}{c|c|c|c|c|c|c}
\hline \multirow{2}{*}{ Modelos } & \multicolumn{3}{|c|}{ Leptospirose } & \multicolumn{3}{c}{ Alagamentos } \\
\cline { 2 - 7 } & EM & REQM & EQEM & EM & REQM & EQEM \\
\hline Matérn $k=0,5$ & 0,013 & 1,236 & 1,527 & 0,127 & 1,311 & 1,719 \\
\hline Matérn $k=1,5$ & $-0,018$ & 1,352 & 1,828 & 0,213 & 1,421 & 2,019 \\
\hline Matérn $k=2,5$ & $-0,027$ & 1,407 & 1,980 & 0,173 & 1,438 & 2,069 \\
\hline Esférico & $-0,052$ & 1,381 & 1,907 & 0,124 & 1,387 & 1,925 \\
\hline Exponencial & 0,013 & 1,236 & 1,527 & 0,127 & 1,311 & 1,719 \\
\hline Gaussiano & $-0,058$ & 1,538 & 2,367 & 0,104 & 1,433 & 2,053 \\
\hline
\end{tabular}
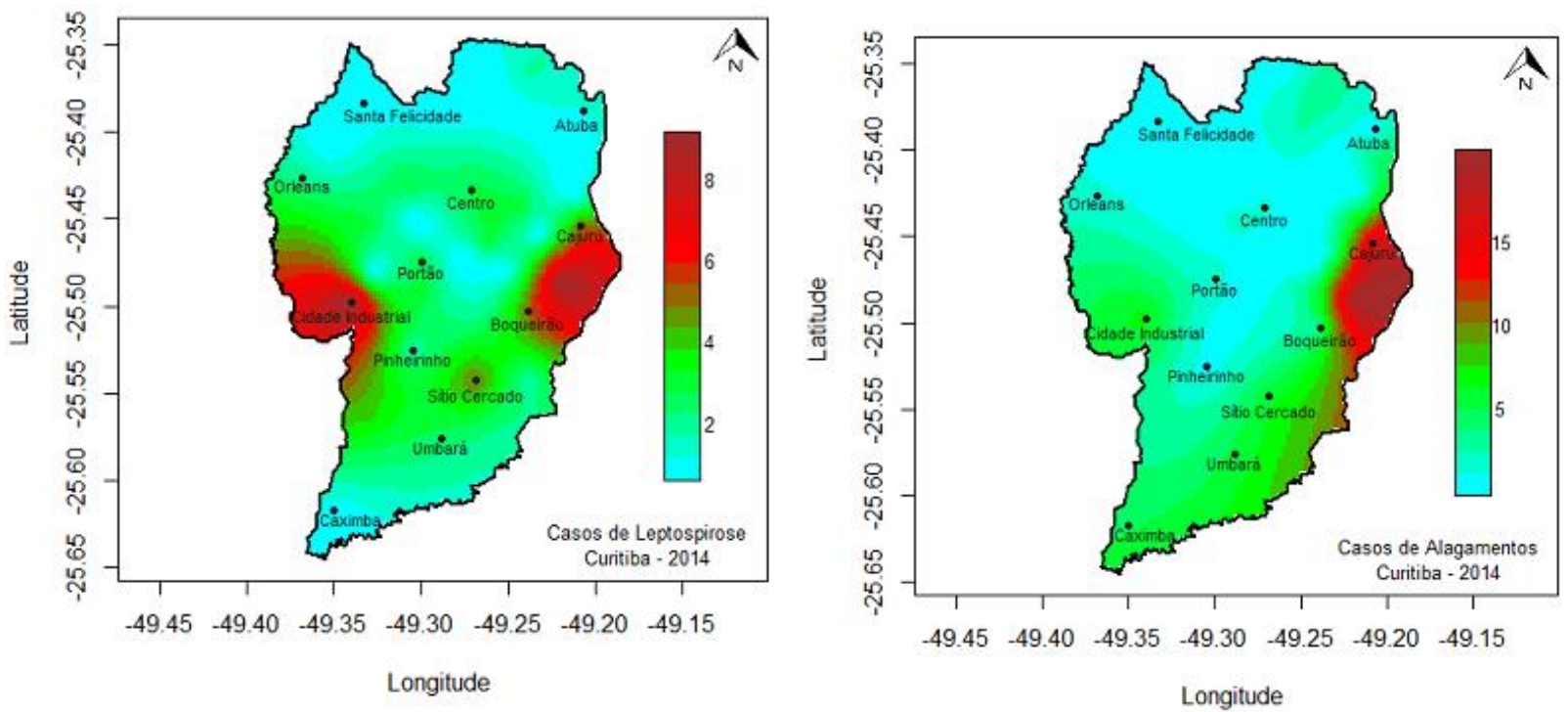

Figura 5: Mapas das estimativas dos casos confirmados de leptospirose (esquerda) e alagamentos (direita) no ano de 2014 no município de Curitiba, gerados a partir dos parâmetros estimados pelo modelo Matérn, $k(0,5)$. 
Após a escolha do modelo realizou-se, então, a krigagem ordinária dos dados utilizando os parâmetros do modelo de semivariograma (Matérn $k=0,5$ ), para estimar a distribuição dos dados confirmados de leptospirose e dos casos de alagamentos no ano de 2014, no município de Curitiba, representados na Figura 5.

Este resultado mostra a situação que se encontrou o município de Curitiba, em relação aos casos confirmados de leptospirose e dos casos de alagamentos no referido ano. Pode ser observado que os bairros com maior número de casos confirmados de leptospirose (Figura 5 (esquerda)) localizados a oeste do município, são os mesmos bairros que apresentam grande incidência de casos de alagamentos (Figura 5 (direita)). No caso da leptospirose há bairros localizados mais a leste do município (Figura 5 (esquerda)) que também apresentaram forte incidência da endemia no ano de 2014. Nos dois casos, leptospirose e regiões de alagamentos, verificou-se que os bairros mais afetados se encontram próximos ao centro do mapa.

Foram construídos os mapas do desvio padrão dos erros das estimativas para os dados de leptos pirose e alagamentos. $O$ desvio padrão dos erros é gerado a partir dos valores da variância da krigagem obtidos juntamente com o cálculo das estimativas. O método para cada estimativa calculada fornece a variância do erro, chamada de variância da krigagem. Pode ser conferida a eficiência da interpolação pelos baixos valores do desvio padrão dos erros das estimativas. Os mapas dos desvios ajustados (Figura 6) mostram que os desvios dos erros são menores próximos dos pontos da amostragem e gradativamente aumentam à medida que se afastam dos pontos amostrais.
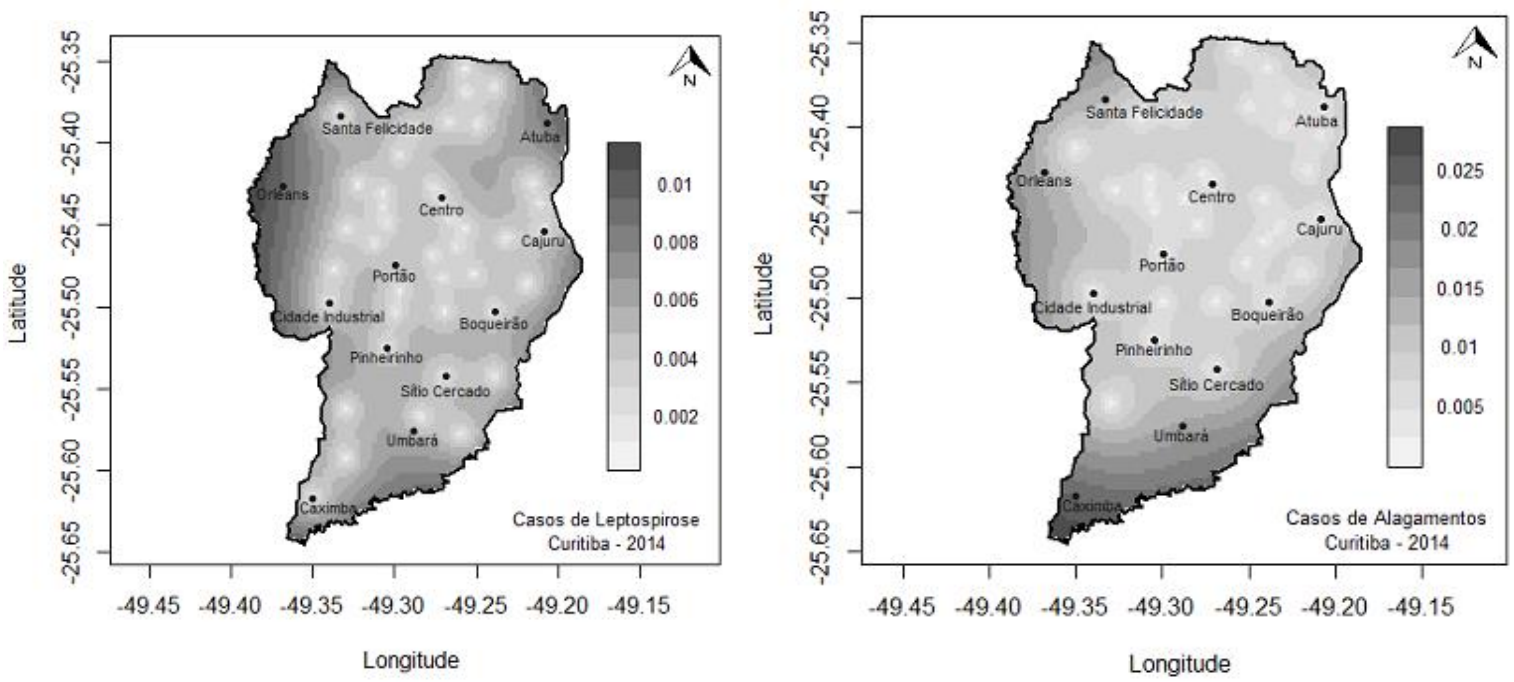

Figura 6: Mapas do desvio padrão dos erros das estimativas dos valores dos casos confirmados de leptospirose (esquerda) e alagamentos (direita) no ano de 2014, no município de Curitiba, gerados a partir dos parâmetros estimados pelo modelo Matérn $k(0,5)$.

O município de Curitiba é dotado de uma densa rede hidrográfica e por isso sofre os impactos das chuvas concentradas de alta intensidade e de curta duração que ocorrem em pequenas áreas. Os impactos observados em Curitiba foram alaga mentos e inundações próximas de rios e córregos, desmoronamentos, danos causados na pavimentação, entre outros, constituindo-se num grande problema para o município (Hayakawa, 2008).

As áreas com maiores casos de alagamentos são próximas aos pontos com casos de leptospirose apresentados neste trabalho. Esses casos encontram-se em pontos próximos aos 
limites de Curitiba, ou seja, nos bairros periféricos. Os mesmos ficam muito próximos aos rios e canais que passam por Curitiba. Geralmente, há moradias em loca is inadequados para a habitação, devido às áreas de várzeas de rios, que estão mais sujeitas às inundações e muitas dessas habitações não possuem acesso a política nacional de saneamento básico. Esses fatores são sugestivos à proliferação da doença.

\section{CONCLUSÃO}

Utilizando a metodologia geoestatística nos dados referentes aos casos confirmados de leptospirose e alagamentos no município de Curitiba no ano de 2014, identificamos a existência de dependência espacial das variáveis. Os resultados obtidos são satisfatórios e levam a crer que o uso desta metodologia traz contribuições significativas para o monitoramento de endemias como essas. A metodologia gera variâncias a cada ponto estimado e com isso foi possível gerar o mapa do desvio padrão das estimativas. Os mapas dos desvios mostram que os desvios dos erros são muito pequenos e isso dá confiabilidade à técnica utilizada.

Os mapas gerados nos mostram a distribuição da endemia nos anos de 2014 e relacionam com as áreas com maiores incidências de alagamentos em Curitiba. Os casos de alagamentos são próximos aos pontos com casos de leptospirose, fator que contribui para a contração da doença. Neste sentido, para o sucesso das ações de planejamento e gestão da saúde, urbana e ambiental, é importante o estabelecimento de mecanismos de monitoramento como este, nas áreas de alagamento recorrentes. Esses procedimentos devem estar associados a políticas habitacionais, que possibilitem o acesso da população de baixa renda a condições digna de moradia, a ações de educação ambiental envolvendo moradores, agentes comunitários e técnicos municipais, além de práticas de planejamento participativo, gestão urbana e ambiental, verdadeiramente democráticas e integradas, com a discussão e troca de informações entre órgãos públicos, setor privado e a sociedade.

\section{REFERÊNCIAS}

1. Albuquerque, M. and MORAIS, H. Descentralization of endemic disease control: an intervention model for combating bancroftian filariasis. Revista Panamericana de Salud Publica/ Pan American Journal of Public Health, 1:155-163, 1997.

2. BERNARDI, IONARA. Leptospirose e saneamento básico. Monografia. Universidade Federal de Santa Catarina. Florianópolis, 2012.

3. BRASIL, MINISTÉRIO DA SAÚDE. Secretaria de Vigilância em Saúde. Guia Leptospirose: Diagnóstico e Manejo Clínico/Ministério da Saúde. Secretaria de Vigilância em Saúde, 2009- no prelo.

4. CAMPOS, JOSMAR FURTADO DE. Eficiência da análise estatística espacial na classificação de famílias do feijoeiro - Estudo via simulação. Dissertação de Mestrado. Universidade Federal de Viçosa. 2011.63p.

5. DIGGLE, P.J., RIBEIRO, P.J.J. Model-based geostatistics. ABE, Caxambu, 2000.

6. DRUCK, S., CARVALHO, M., CÂMARA, G., MONTEIRO, A. Análise Espacial de Dados Geográficos. EMBRAPA, 2004. 
7. HAYAKAWA, IURI F.; ULTRAMAR, CLOVIS. Situações de risco como definidoras de inflexões no planejamento e na gestão urbana: um estudo em Curitiba. Trabalho apresentado no XVI Encontro Nacional de Estudos Populacionais, outubro de 2008

8. ISAAKS, E.H., SRIVASTAVA, R.M., (1989). Applied Geostatistics. Oxf University, New York, 561p.

9. LANDIM, P.M.B. \& STURARO, J.R. Krigagem indicativa aplicada à elaboração de mapas probabilísticos de riscos. DGA,IGCE,UNESP/Rio Claro, Lab. Geomatemática,Texto Didático 06, 19 pp. 2002.

10. LIMA, RENAN CHAVES DE. Um estudo epidemiológico e aplicação de medidas preventivas em uma região do município de Belém, Pará. Trabalho de Conclusão de Curso. Faculdade de Biomedicina da Universidade Federal do Pará. 2009. 76p.

11. LOHMANN, MARCIEL. Análise dos alagamentos no município de Curitiba entre os anos de 2005 a 2010. Artigo publicado em julho de 2013, Ciência Geográfica, Bauru - XVII. Vol. XVII.

12. MONEGO, MAURICI LUZIA DEL. Aplicação de Métodos Geoestatísticos na Modelação e Caracterização de Descargas de Águas Residuais. Tese de doutorado. Faculdade de Engenharia da Universidade do Porto, Portugal. 2012. 166p.

13. OLIVEIRA, MARIA CRISTINA NEVES DE. Métodos de estimação de parâmetros em modelos geoestatísticos com diferentes estruturas de covariâncias: Uma aplicação ao teor de cálcio no solo. Tese para obtenção de título de Doutor. São Paulo, Janeiro 2003.

14. PAZ-GONZALEZ, A., TABOADA CASTRO, M.T., VIEIRA, S.R. Geostatistical analysis of heavy metals in a one-hectare plot under natural vegetation in a serpentine area. Canadian Journal of Soil Science, vol. 81:pp. 469_479, 2001.

15. R DEVELOPMENT CORE TEAM. R: A Language and Environment for Statistical Computing. R Foundation for Statistical Computing, Vienna, Austria, 2006. ISBN 3-900051-07-0.

16. RIBEIRO JR., P., DIGGLE, P. _geoR: a package for geostatistical analysis_. RNEWS, vol. 1, 2:pp. 15_18, 2001.

17. SARKAR, U., NASCIMENTO, S., BARBOSA, R., MARTINS, R., NUEVO, H., KALAFANOS, I., GRUNSTEIN, I., FLANNERY, B., DIAS, J., RILEY, L., REIS, M. AND KO, A. Population based casecontrol investigation of risk factors for leptospirosis during urban epidemic. American Journal of Tropical Medicine and Hygiene, 66:605-6100, 2002.

18. SOARES, A. (2000). Geoestatística para as Ciências da Terra e do Ambiente, IST Press, Lisboa.

19. TASSINARI, WAGNER DE SOUZA. Modelagem espacial, temporal e longitudinal: diferentes abordagens do estudo da leptospirose urbana. Tese para obtenção de título de Doutor. Rio de Janeiro, Março de 2009.

20. WACKERNAGEL, H. Multivariate Geostatistics: an introduction with applications. Springer, 3rd ed., 2003.

21. WEI, H., DAI, L., WANG, L. (2007). Spatial distribution and risk assessment of radionuclides in soils around a coalfired power plant: A case study from the city of Baoji, China. Environmental Research, 104, pp. 201-208. 